\title{
A SHORT PROOF THAT METRIC SPACES ARE REALCOMPACT ${ }^{1}$
}

\author{
M. D. RICE
}

\begin{abstract}
A short proof is given that metric spaces which have no closed discrete subspaces of measurable cardinal are realcompact. This result is used to obtain the Shirota theorem that topologically complete spaces which have no closed discrete subspaces of measurable cardinal are realcompact.
\end{abstract}

The result stated in the title is usually obtained as a corollary to the Katětov theorem [3] that paracompact spaces with certain cardinal restrictions are realcompact, or the Shirota theorem [5] mentioned in the abstract. Both my proof and Katětov's proof use techniques similar to those used by Marczewski-Sikorski [4], Theorem 6, which implicitly contains a proof of the following theorem due to Stone [6].

THEOREM 1. Every open cover of a metric space has a $\sigma$-discrete open refinement.

For completeness we sketch the proof. Let $\mathscr{A}=\left\{A_{s}\right\}_{s \in S}$ be an open cover of the metric space $(X, d)$. Let $A_{s, n}=\left\{x: d\left(x, X-A_{s}\right)>1 / 2^{n}\right\}$ for $s \in S$ and $n \in N$. These are clearly open sets. Now suppose $<$ is a well-ordering of $S$ and define $B_{t, n}=A_{t, n}-\operatorname{cl}\left(\bigcup_{s<t} A_{s, n+1}\right)$. Then $\mathscr{B}_{n}=\left\{B_{t, n}\right\}_{t \in S}$ is a discrete family of open sets and $\mathscr{B}=\bigcup_{n \in N} \mathscr{B}_{n}$ is a $\sigma$-discrete open refinement of $\mathscr{A}$.

For the terminology used below we refer the reader to Chapters 8 and 12 of [2].

THEOREM 2. Let $X$ be a metric space which has no closed discrete subspace of measurable cardinal. Then $X$ is realcompact.

If $X$ is not realcompact, there exists a free ultrafilter of zero sets $\mathscr{F}$ with the countable intersection property. Let $\mathscr{A}=\{\operatorname{Int} Z(f): Z(f) \notin \mathscr{F}\}$. We easily see that $\mathscr{A}$ is an open cover of $X$, so let $\mathscr{B}=\bigcup \mathscr{B}_{n}$ be a $\sigma$-discrete open refinement of $\mathscr{A}$. Setting $A_{n}=\bigcup\left\{E: E \in \mathscr{B}_{n}\right\}$, we have $\bigcap\left(X-A_{n}\right)=$ $\varnothing$, which shows $X-A_{m} \notin \mathscr{F}$ for some $m \in N$ since $\mathscr{F}$ has the countable intersection property. Thus $X-A_{m} \cup \bar{A}_{m} \in \mathscr{F}$ implies $\bar{A}_{m} \in \mathscr{F}$. Define $\mu: \mathscr{P}\left(\mathscr{B}_{m}\right) \rightarrow\{0,1\}$ by $\mu(\mathscr{S})=1$ if $\operatorname{cl}(\bigcup \mathscr{S}) \in \mathscr{F} ; \mu(\mathscr{S})=0$ if $\operatorname{cl}(\bigcup \mathscr{S}) \notin \mathscr{F}$.

Received by the editors February 1, 1971.

AMS 1970 subject classifications. Primary 54D60, 54-02; Secondary 54E15.

Key words and phrases. Realcompact, topologically complete, ultrafilter, measurable cardinal.

${ }^{1}$ Research partially supported by NSF Grant GP-18825.

(c) American Mathematical Society 1972 
Then $\mu$ is a measure since $\mathscr{B}_{m}$ is a discrete family and $\mu\left(\mathscr{B}_{m}\right)=1$ since $\bar{A}_{m} \in \mathscr{F}$. From the definition of $\mathscr{A}$ we see that $\mu\{E\}=0$ for $E \in \mathscr{B}_{m}$. Thus $\mathscr{B}_{m}$ has measurable cardinal. Choosing one point from each member of $\mathscr{B}_{m}$ gives a closed discrete subspace of $X$ with measurable cardinal.

Finally, define $X$ to be topologically complete if for each $p \in \beta X-X$ there exists a continuous function $f_{p}: X \rightarrow M_{p}$, where $M_{p}$ is a metric space, which has no continuous extension to $p$. [Here $\beta X$ denotes the Stone-Čech compactification of $X$.] Suppose $X$ is topologically complete and has no closed discrete subspace of measurable cardinal. Using Theorem 2 we easily show that $X$ is realcompact. ${ }^{3}$ For $p \in \beta X-X$, let $f_{p}: X \rightarrow M_{p}$ be as described above. Then no closed discrete subspace of $f_{p}[X]$ has measurable cardinal; hence, by Theorem $2, f_{p}[X]$ is realcompact. Let $g_{p}: \nu X \rightarrow f_{p}[X]$ denote the extension of $f_{p}$ over $v X$, the Hewitt-realcompactification of $X$. Then, by definition of $f_{p}, v X \subseteq \beta X-\{p\}$ for each $p \in \beta X-X$; hence $X=v X$ and $X$ is realcompact. Conversely, since $\nu X$ is the unique realcompact space in which $X$ is dense and $C$-embedded, realcompactness implies topological completeness. [For $p \in \beta X-X$, there exists a suitable function from $X$ into the real line.]

\title{
REFERENCES
}

1. R. Engelking, Outline of general topology, PWN, Warsaw, 1965; English transl., North-Holland, Amsterdam; Interscience, New York, 1968. MR 36 \#4508; MR 37 \#5836.

2. L. Gillman and M. Jerison, Rings of continuous functions, University Series in Higher Math., Van Nostrand, Princeton, N.J., 1960. MR 22 \#6994.

3. M. Katětov, Measures in fully normal spaces, Fund. Math. 38 (1951), 73-84. MR 14, 27.

4. E. Marczewski and R. Sikorski, Measures in non-separable metric spaces, Colloq. Math. 1 (1948), 133-139. MR 10, 23.

5. T. Shirota, A class of topological spaces, Osaka Math. J. 4 (1952), 23-40. MR 14, 395.

6. A. H. Stone, Paracompactness and product spaces, Bull. Amer. Math. Soc. 54 (1948), 977-982. MR 10, 204.

\author{
Department of Mathematics, Wesleyan University, Middletown, Connecticut \\ 06457
}

\footnotetext{
${ }^{2}$ This definition of topological completeness is equivalent to $X$ admitting a compatible uniformity which is complete, using the following result which was pointed out to me by $A$. W. Hager.

THEOREM. Let $\mu X$ be a uniform space which is weakly generated by the family of functions $\left\{f: X \rightarrow \rho_{f} M_{f} \mid f \in \mathscr{F}\right\}$, where $\rho_{f} M_{f}$ is a complete metric space and $f[X]$ is dense in $M_{f}$. If $\hat{f}$ denotes the extension of $f$ to the Samuel compactification $\beta \mu X$ of $\mu X$, with range $\beta \rho_{f} M_{f}$, then $Z=\bigcap\left\{\hat{f}^{-1}[f[X]]: f \in \mathscr{F}\right\}$ with the weak uniformity generated by $\left\{\hat{f}_{\mid Z}: Z \rightarrow \rho_{f} M_{f} \mid f \in \mathscr{F}\right\}$ is the completion of $\mu X$.

${ }^{3}$ This was pointed out to me by A. W. Hager.
} 